\section{Protocol of an Observational, Follow up, Single Arm Study for Knowing the Compliance of Patients Scheduled for Major Abdominal and Thoracic Surgeries Who Were Instructed to Perform Spirometry in a Single Specialty Centre}

\begin{abstract}
Abhijit S. Nair ${ }^{1 *}$, Suresh Seelam ${ }^{1}$, Aanchal Bharuka ${ }^{1}$, Priya P. Nayak ${ }^{1}$, Mohammad Salman Saifuddin ${ }^{1}$, Basanth Kumar Rayani ${ }^{1}$

${ }^{1}$ Department of Anaesthesiology, Basavatarakam Indo-American Cancer Hospital and Research Institute, Road No.10, Banjara Hills, Hyderabad-500034, Telangana State, India.
\end{abstract}

Corresponding to: Abhijit S. Nair. Email: abhijitnair95@gmail.com .

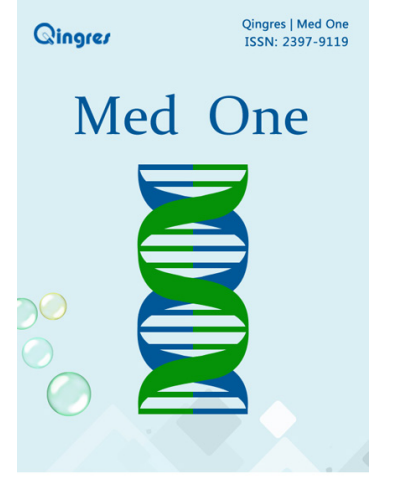

\section{STUDY PROTOCOL}

Protocol of an observational, follow up, single arm study for knowing the compliance of patients scheduled for major abdominal and thoracic surgeries who were instructed to perform spirometry in a single specialty centre.

\begin{abstract}
Peri-operative incentive spirometry (IS) helps in improving pulmonary function, facilitates sputum clearance and prevents unwanted postoperative pulmonary complications after major abdominal and thoracic surgery. In our hospital, all patients are instructed to practice IS before abdominal and thoracic surgeries so that they can perform it in the postoperative period effectively. However, many patients do not follow our advice. A few unfortunate patients land up with pulmonary complications as it becomes difficult to train them after surgery.
\end{abstract}

\section{Methods and analysis}

We designed an observational, follow up, single arm study to know the 
reason of non-compliance so as to educate them in the best possible way regarding optimal use of IS in the preoperative period. The patients would be instructed to perform IS in front of accompanying relatives and the effort will be noted. They would be provided with an information leaflet in the language that they understand well. The IS effort and the technique of performing IS would be noted on the day of surgery. The patients performing correctly would be labelled as compliant. The ones who are performing IS incorrectly and ineffectively would be labelled as non-compliant. The patient and family members would be asked questions to know the cause of non-compliance. The answers obtained would guide in educating the patients more effectively in future so as to have better compliance and postoperative outcomes.

\section{Trial registration}

The trial is registered with Clinical Trials Registry of India (CTRI). The registration number is CTRI/2017/03/008122, registered on 16/3/2017.

Keywords: Incentive spirometry; postoperative pulmonary complications; patient education; surgery, infection

\section{INTRODUCTION}

Preoperative incentive spirometry (IS) is useful in patients who are scheduled for major abdominal surgeries and thoracic surgeries. It helps them by improving the lung function and facilitates sputum clearance. Another reason of starting the exercises in the preoperative period is to educate the patients so that they can continue doing IS in the postoperative period effectively. By doing so, the possibility of postoperative pulmonary complications (POPC) would be less due to better sputum clearance and adequate lung expansion. In our hospital, the anaesthesiologist advices IS at the time of pre-anaesthesia check up (PAC) to all patients who are scheduled for major abdominal and thoracic surgeries. The patient is instructed by the anaesthesiologist and the respiratory therapist in the PAC clinic in front of family members who accompany the patients. They are also informed in detail about the benefits of performing IS in the postoperative period. A printed leaflet is given to them in the language that they understand that describes the detailed technique of how to do and what not to do. The fitness for surgery is given in most of the situations only if the patient is performing spirometry well. In spite of this, on many occasions we have observed that the same patient is unable to perform spirometry as instructed in the preoperative period. We thought the reason could be poor compliance in performing spirometry at home as instructed, lack of family support, inability to understand the importance of IS or the technique. We therefore decided to conduct an observational, follow up, single arm trial to know the compliance rate among these group of patients by noting the spirometry performance in ' $m l$ ' preoperatively which is printed on the incentive spirometer equipment. This value is compared to the value achieved on the day of surgery. With regular practice, the performance has to either improve or at least remains the same. But in patients whose performance is below that of the preoperative levels or patients who are not performing it in the way as told to them, we shall try to analyse the cause of non-compliance. We shall try to find out the cause of poor compliance by asking few questions to the patient and the family members.

\section{PROTOCOL}

\subsection{Review of literature}

Commonly observed POPCs are atelectasis, pneumonia and hypoxic respiratory failure. According to data published by Fisher et $a{ }^{\left[{ }^{[1]}\right.}$ and by Gatti et $\mathrm{al}^{[2]}$ the incidence of pulmonary complications postoperatively is in the range of 2-39\%. Upper abdominal surgeries, thoracic surgeries, lower abdominal surgeries are responsible for these situations. IS, deep breathing exercises, directed coughing, early mobilization, and optimal analgesia can help in preventing or reducing the severity of POPCs. Incentive spirometry is similar to a sigh or yawning. This is known to decrease pleural pressure, promote better lung expansion, facilitate mucociliary clearance and helps in better gas exchange. If IS is performed regularly using the correct technique, it can reduce the possibility of POPCs. This was highlighted by the study published by Westwood et $a l^{[3]}$. Kundra et al randomised 50 patients who were scheduled for a laparoscopic cholecystectomy under general anaesthesia ${ }^{[4]}$. First group was the control who were not advised preoperative IS. The second group of patients practised IS for a week. They recorded lung functions during PAC, on the day before the surgery, postoperatively at 6,24 and 48 hours and at the time of discharge in both groups. They found that lung functions were better preserved 
in patients who practised IS preoperatively. Kulkarni et al randomised 80 patients undergoing major abdominal surgery in 4 groups viz. control, deep breathing exercises, IS and inspiratory muscle training groups ${ }^{[5]}$. The patients in group 2,3,4 were advised to perform the allotted exercises for 2 weeks prior to surgery. They found that patients who were in the inspiratory muscle training group had better and preserved lung functions than other groups. However, IS has not been shown to be beneficial in patients undergoing coronary artery bypass grafting (CABGs) and bariatric surgery ${ }^{[6-8]}$. Junior et al has mentioned in Cochrane Database of Systematic Reviews that there is low quality evidence to support the regular use of IS in preventing postoperative pulmonary complications ${ }^{[9]}$. However, the available literature has not described patient compliance when IS was started preoperatively. Adequately powered and well designed studies are required to arrive at a conclusion. Along with IS, patients should be advised to perform deep breathing exercises. Although Restrepo et al felt that IS should not be routinely used preoperatively to prevent atelectasis; a volume oriented IS device with deep breathing exercises, early mobilization and optimal postoperative analgesia can help in reducing the severity of POPC [10].

\subsection{Ethics committee approval}

We have taken approval from the hospital Ethics Committee to conduct this observational study (EC
Reference No. IEC/2017/28, dated 23/02/2017).

\subsection{Study registration}

We registered our study with Clinical Trials Registry of India (CTRI). The registration number is CTRI/2017/03/008122, registered on 16/3/2017.

\subsection{Study design}

Our study is an observational, follow up, single arm trial registered with CTRI. The intent of the study is to find out reason for non-compliance among surgical patients who were instructed to perform IS so as to prevent pulmonary complications in the postoperative period. We prepared our protocol using the SPIRIT (Standard Protocol Items for Randomized Trials) recommendations (http://www.spiritstatement.org/) [11]. The patients will be provided with an information leaflet which will tell them the do's and don'ts while using a spirometer. On the day of the surgery, we will review the spirometric effort in 'ml', the way of holding the mouth piece of the instrument and the way of performing IS. If it is found satisfactory, the patient shall be declared compliant and no further questions will be asked. However, if the patient is not able to hold the mouthpiece properly or perform breathing exercise adequately, the patient would be considered non-compliant. We would ask the patient and family members a set of questions to know the reason of non-compliance. (Table 1) A flow diagram based on the CONSORT (Consolidated Standards of Reporting Trials) statement is shown in Fig. 1.

Table 1. Questions which would be asked to the non-compliant patients and the family members.

Did you understand the instructions?

Did you have time to practice?

Did your family members help you in practising?

Did you help the patient in understanding and performing spirometry?

Did you have time for the patient? 


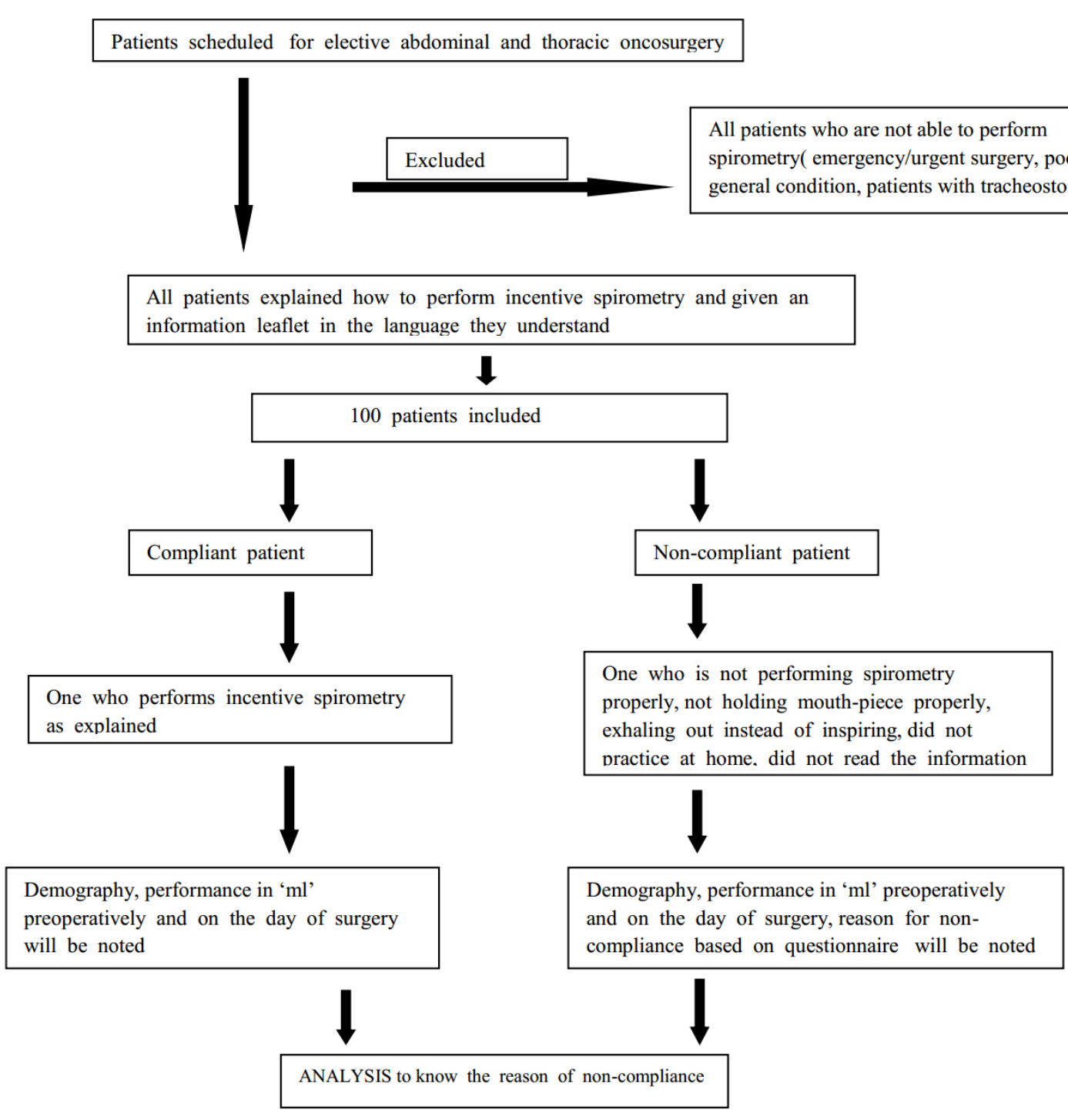

Fig. 1 Flow-chart of this study

\subsection{Place of study}

The study will be conducted by the Department of Anaesthesiology at Basavatarakam Indo-American Cancer Hospital and Research Institute (BIACH and $\mathrm{RI})$ which is a 500 bedded single speciality, Cancer Hospital at Hyderabad, India. The first part of the study would be at the PAC clinic where patient will be recruited in the study. The second part would be in the preoperative holding area where the compliance status would be determined and if non-compliant, the questions would be asked.

\subsection{Recruitment (inclusion and exclusion criteria)}

We shall recruit all patients who are scheduled to undergo major abdominal and thoracic surgeries. We shall stop recruiting once we complete 100 recruitments. One hundred patients will be recruited and analysed in the order of arrival in the PAC Clinic. Patients coming for emergency and urgent surgeries will not be recruited. Patients with preexisting tracheostomy, on home continuous positive airway pressure (CPAP) machine, having intercostals drains (ICDs) in situ will be excluded from the study.

\subsection{Type of surgeries}

The type of abdominal and thoracic surgeries that are done in this hospital is shown in a tabular form in Table 2 (a) and 2 (b). 
Table 2 (a). The abdominal surgeries performed in our hospital.

S.no. Abdominal surgery

$\begin{array}{ll}1 & \text { Interval cytoreduction( for carcinoma ovary) } \\ 2 & \text { Radical hysterectomy } \\ 3 & \text { Gastrectomy } \\ 4 & \text { Hemicolectomy } \\ 5 & \text { Whipple's procedure } \\ 6 & \text { Radical cystectomy } \\ 7 & \text { Radical cholecystectomy } \\ 8 & \text { Total colectomy } \\ 9 & \text { Nephrectomy } \\ 10 & \text { Hepatectomy } \\ 11 & \text { Trans-hiatal oesophagectomy }\end{array}$

Table 2 (b). The thoracic surgeries performed in our hospital

\section{S.no. Thoracic surgery}

\begin{tabular}{ll}
\hline 1 & Lobectomy \\
2 & Pneumonectomy \\
3 & Transthoracic oesophagectomy \\
4 & Mediastinal tumor excision \\
5 & Chest wall tumor excision \\
6 & Thymoma excision \\
\hline
\end{tabular}

\subsection{Randomisation}

There is no randomisation which will be followed. One hundred patients will be recruited in the study in the order of arrival in the PAC clinic, fulfilling inclusion criteria and not having any of the exclusion criteria.

\subsection{Intervention}

This is an observational study and has no interventions involved.

\subsection{Data collection}

The details will be entered in an Excel sheet which will be analysed later. We will be noting age, gender, type of surgery, IS effort in $\mathrm{ml}$ (2 values): 1st value i.e. the one that was observed in PAC clinic and 2 nd value i.e. observed immediately prior to surgery. The answers to the questions in the form of 'YES' and 'NO' will also be entered in the Excel sheet.

\section{11 Data analysis}

A biostatistician will be consulted for the analysis of data collected using appropriate statistical methods. The independent variables will be compared using the Mann-Whitney test and Student's t-test. The proportions will be compared using Fisher's exact test.

\section{12 Publication plan and dissemination}

Once we finish recruitment and complete analysis to find answers to our questions, we shall plan to write it as an observational study and submit the manuscript to peer reviewed journal. Anybody who is interested to know more about the study could contact the corresponding author or the principal investigator via email. We would award authorships based on the criteria described by the International Committee of Medical Journal Editors (ICJME) ${ }^{[12]}$.

\section{DISCUSSION}

Although preoperative use of IS before major surgery has equivocal benefits, a patient who knows how to use it before hand can use it effectively in the postoperative period. On analysis of the answers to the questions, we might get the reasons why our patients were non-compliant in performing IS in spite of explaining in detail in the preoperative period and also providing them with a leaflet. We could then use those answers in developing a better way of instructing the patients before major thoracic and abdominal surgeries so as to prevent POPCs. Another question that arises is that are POPCs more or the same in non-compliant patients when compared to the otherwise compliant patient. This could be answered by prospectively following these patients till discharge or till 30 days after surgery whichever is earlier. Several factors that could lead to POPCs in the compliant as well as non-compliant 
patients are inadequate pain relief, presence of ICDs postoperatively, poor general condition like hypoalbuminemia, body mass index $<15 \mathrm{~kg} / \mathrm{m} 2$, acute lung injury (aspiration, transfusion, ventilator associated, use of one lung ventilation intraoperatively).

\section{CONFLICT OF INTERESTS}

Authors claim no conflict of interests.

\section{REFERENCES}

1. Fisher BW, Majumdar SR, McAlister FA. Predicting pulmonary complications after nonthoracic surgery: a systematic review of blinded studies. Am J Med. 2002;112(3):219225.

2. Gatti G, Cardu G, Lusa AM, Pugliese P. Predictors of postoperative complications in high-risk octogenarians undergoing cardiac operations. Ann Thorac Surg. 2002;74(3):671677.

3. Westwood K, Griffin M, Roberts K, Williams $M$, Yoong K, Digger T. Incentive spirometry decreases respiratory complications following major abdominal surgery. Surgeon. 2007;5(6):339-342.

4. Kundra P, Vitheeswaran M, Nagappa M, Sistla S. Effect of preoperative and postoperative incentive spirometry on lung functions after laparoscopic cholecystectomy. Surg Laparosc Endosc Percutan Tech. 2010;20(3):170-172.

5. Kulkarni S, Fletcher E, McConnell A, Poskitt K, Whyman M. Pre-operative inspiratory muscle training preserves postoperative inspiratory muscle strength following major abdominal surgery - a randomised pilot study. Ann R Coll Surg Engl. 2010;92(8):700-705.

6. Bergin C, Speroni KG, Travis T, Bergin J, Sheridan MJ, Kelly K, Daniel MG. Effect of preoperative incentive spirometry patient education on patient outcomes in the knee and hip joint replacement population. J Perianesth Nurs. 2014;29(1):207.

7. Clavellina-Gaytán $D$, Velázquez-Fernández $D$, Del-Villar E, Domínguez-Cherit G, Sánchez H, Mosti M, Herrera MF. Evaluation of spirometric testing as a routine preoperative assessment in patients undergoing bariatric surgery. Obes Surg. 2015;25(3):530-536.

8. Cattano D, Altamirano A, Vannucci A, Melnikov V, Cone C, Hagberg CA.Preoperative use of incentive spirometry does not affect postoperative lung function in bariatric surgery. Transl Res. 2010;156(5):265-272.

9. do Nascimento Junior P, Módolo NSP, Andrade S, Guimarães MMF, Braz LG, El Dib R. Incentive spirometry for prevention of postoperative pulmonary complications in upper abdominal surgery. Cochrane Database Syst Rev. 2014; 2(2): CD006058.

10. Restrepo RD, Wettstein R, Wittnebel L, Tracy M. Incentive spirometry: 2011. Respir Care. 2011;56(10):1600-1604.

11. Chan AW, Tetzlaff JM, Gøtzsche PC, Altman DG, Mann H, Berlin JA, Dickersin K, Hróbjartsson A, Schulz KF, Parulekar WR, Krleza-Jeric K, Laupacis A, Moher D. SPIRIT 2013 explanation and elaboration: guidance for protocols of clinical trials. BMJ. 2013;346:e7586.

12. International Committee of Medical Journal Editors. Recommendations for the conduct, reporting, editing, and publication of scholarly work in medical journals. Updated December 2015. http://www.icmje.org/ icmjerecommendations. 